\title{
Transplantation of HCV Viremic Livers into HCV Viremic Recipients Followed by Direct-acting Antiviral Therapy
}

\author{
Nikhil Kapila ${ }^{1}$, Kawtar Al Khalloufi ${ }^{2}$, Gianina Flocco ${ }^{3}$, K.V. Narayanan Menon ${ }^{3}$, \\ Christina Lindenmeyer ${ }^{3}$, Diego Reino ${ }^{2}$, Jason M. Vanatta ${ }^{2}$, Samer Ebaid², Andreas Tzakis ${ }^{2}$ \\ and Xaralambos Bobby Zervos*2
}

${ }^{1}$ Department of Gastroenterology and Hepatology, Cleveland Clinic Florida, Weston, FL, USA; ${ }^{2}$ Department of Transplant, Cleveland Clinic Florida, Weston, FL, USA; ${ }^{3}$ Department of Gastroenterology and Hepatology, Cleveland Clinic, Cleveland, $\mathrm{OH}$, USA

\begin{abstract}
Background and Aims: Hepatitis C virus (HCV)-infected organs are underutilized. We aimed to assess the safety and efficacy of direct-acting antiviral agents (DAAs) therapy in $\mathrm{HCV}$ viremic patients who are transplanted with a liver from a HCV viremic donor. Methods: We conducted a retrospective study, including patients seen from July 2015 to April 2017. $\mathrm{HCV}$ viremic patients transplanted with a liver from a HCV viremic donor and subsequently treated with DAAs were included. Outcomes assessed included undetectable viral load at 12 weeks after completing DAA therapy (sustained virologic response, $\mathrm{SVR}_{12}$ ), adverse events, and interactions with immunosuppression. Results: Twenty-four HCV viremic recipients received livers from HCV viremic donors. Median age was 63 years, and the majority (79.2\%) were genotype 1 a. Donors and recipients were viremic at the time of transplant. Median modified model for end-stage liver disease score was 19 , and median time on the waitlist was 81 days. Median time from transplant to initiation of DAA therapy was 123 days. Several DAA regimens were used and 15 (62.5\%) patients did not receive ribavirin. Treatment duration ranged from 12 to 24 weeks. Twenty-three $(95.8 \%)$ patients achieved SVR 12 . Five $(20.8 \%)$ patients developed adverse events; however, none required DAA discontinuation. Conclusions: DAA therapy was efficacious and well tolerated in $\mathrm{HCV}$ viremic recipients who underwent liver transplantation from a HCV viremic donor. Citation of this article: Kapila N, Khalloufi KA, Flocco G, Menon KVN, Lindenmeyer $C$, Reino $D$, et al. Transplantation of $\mathrm{HCV}$ viremic livers into $\mathrm{HCV}$ viremic recipients followed by direct-acting antiviral therapy. J Clin Transl Hepatol 2019;7(2):122-126. doi: 10.14218/JCTH.2019.00014.
\end{abstract}

\section{Introduction}

Cirrhosis secondary to hepatitis C virus (HCV) is the second most common indication for liver transplantation (LT) in the

Keywords: Hepatitis C; Direct-acting antiviral agents; Donor pool expansion; High risk donors; Liver transplantation.

Abbreviations: DAA, direct-acting antiviral agents; HCV, hepatitis C virus; LT, liver transplantation; MELD-Na, modified model for end-stage liver disease; SVR, sustained virologic response.

Received: 27 March 2019; Revised: 26 April 2019; Accepted: 15 May 2019

*Correspondence to: Xaralambos Bobby Zervos, Department of Transplant, Cleveland Clinic Florida, 2950 Cleveland Clinic Boulevard, Weston, FL 33331 USA. Tel: +1-954-659-5133, Fax: +1-954-659-6731, E-mail: zervosx@ccf.org
USA. ${ }^{1}$ Recurrence of infection in the donor graft is essentially universal if HCV RNA is detectable in the recipient prior to LT. The course of HCV in the immunosuppressed transplant recipient is more aggressive than that in immunocompetent individuals, resulting in rapid development of fibrosis, with approximately $30 \%$ of patients developing cirrhosis within 5 years of transplantation. ${ }^{2,3}$

Approximately $50 \%$ of candidates listed for liver transplant in the USA in 2012 waited more than 3 years to receive an organ. Despite the significant waiting time, HCV-positive grafts are almost twice as likely to be discarded compared to HCV-negative grafts. ${ }^{4}$ While over $25 \%$ of patients listed for liver transplant have a diagnosis of $\mathrm{HCV}$, recent data indicates that only $4.4 \%$ of these potential recipients receive an HCVpositive graft. ${ }^{1}$

In the past, the management of HCV in the posttransplant population was limited to the combination of interferon and ribavirin. ${ }^{5}$ This regimen was far from ideal, considering the low tolerability and suboptimal response rates. HCV treatment has evolved since the introduction of direct-acting antiviral agents (DAAs) ${ }^{6,7}$ In 2014, the second generation of DAAs were introduced and a paradigm shift was noted in the management of $\mathrm{HCV}^{8}$ The use of these interferon-free regimens has proven to be effective in the general population, ${ }^{9}$ and recent clinical trials have suggested that these agents, with or without ribavirin, are efficacious and well-tolerated post LT. ${ }^{10-13}$ The advent of effective and well-tolerated antiviral regimens allows healthcare providers to not only deliver effective post-LT therapy but also allows for potential expansion of the donor pool. ${ }^{14}$ Limited data exists on the utilization of grafts from HCV viremic donors in HCV viremic recipients in the era of DAA therapy. ${ }^{15}$

The aim of this study was to report our experience with the use of DAAs in HCV viremic patients who underwent LT with a graft from a HCV viremic donor.

\section{Methods}

After receiving approval from the Institutional Review Board, a multicenter, retrospective chart review was conducted. The electronic medical record was reviewed to include HCVpositive patients who underwent LT with an HCV-positive graft, and were subsequently treated with DAAs. Both donor and recipient were viremic at the time of transplant, as determined by HCV nucleic acid amplification testing. 
A set of variables was extracted from the medical record. These included basic demographic data and HCV genotype. $\mathrm{HCV}$ viral loads were determined prior to initiation of therapy, as well as at set intervals during and after completion of treatment. A second group of biochemical parameters studied included pre- and post-treatment values for alanine transaminase, total bilirubin, glomerular filtration rate, albumin, and platelet count. Liver fibrosis prior to treatment was assessed by review of histology from liver biopsy, and if liver biopsy was not available, by calculating a fibrosis-4 score. Liver fibrosis was not objectively measured during treatment.

The antiviral regimen, duration of treatment, and timing of therapy were determined by the prescribing hepatologist based on a variety of factors, including posttransplant genotype, prior treatment, degree of graft fibrosis, and renal function. The antiviral regimen consisted of DAAs with or without use of ribavirin.

$\mathrm{HCV}$ viral load was quantified using HCV polymerase chain reaction. The lower limit of quantification was $15 \mathrm{IU} / \mathrm{mL}$. These levels were monitored at baseline, at completion of treatment, and then at 12 weeks following treatment in order to determine sustained virologic response (SVR). The primary end-point was the proportion of patients with undetectable viral loads at 12 weeks after completing treatment $\left(\mathrm{SVR}_{12}\right)$.

Data on adverse events was extracted from the medical record after reviewing laboratory values and progress notes. Rejection was characterized by histological evidence of rejection or elevated liver biochemistries that responded to adjustment in systemic immunosuppression.

\section{Results}

Between July 2015 and April 2017, 24 HCV viremic patients underwent LT with a graft from a HCV viremic donor and were subsequently treated with a DAA-based regimen. Of these 24 patients, 5 underwent a combined liver-kidney transplant. The baseline characteristics of the donors and recipients are shown in Table 1 . The majority of the cohort was male (67\%) and infected with genotype 1a (79.2\%). Three patients $(12.5 \%)$ had a different HCV genotype after transplant. Pretransplant, one patient was genotype $1 \mathrm{a}$ and converted to genotype 3 after transplant, another patient converted from $1 \mathrm{~b}$ to $1 \mathrm{a}$, and the last patient transitioned from genotype 4 to 1 a. The median age was 63 years. The median modified model for end-stage liver disease (MELD-Na) score at the time of transplant was 19 (range: 14-35), median time on the transplant waitlist was 81 days (range: 4-397), and median time from transplant to initiation of antiviral therapy was 123 days (range: $10-350)$. Eleven patients (45.8\%) were treatment naïve at the time of $\mathrm{LT}$, two patients $(8.3 \%)$ had previous experience with DAAs, and the remainder $(45.8 \%)$ had been unsuccessfully treated with interferonbased regimens.

The donors in our cohort were viremic at the time of harvesting the organ. Pathology from frozen sections done prior to DAA treatment was available for 17 patients. Thirteen patients had no fibrosis, three patients had F1 fibrosis, and one patient had F2 fibrosis. Seven patients did not have a liver biopsy available, however the median fibrosis- 4 score of these patients was 2.07. Treatment regimen and duration were determined by the individual hepatologist and are listed in Table 2. All patients had a glomerular filtration rate of greater than $30 \mathrm{~mL} / \mathrm{min} / 1.73 \mathrm{~m}^{2}$ at the time of starting treatment. Nine patients received regimens that included ribavirin. The doses of
Table 1. Baseline demographics and clinical characteristics

\begin{tabular}{|c|c|c|}
\hline Recipient characteristic & & IQR \\
\hline Age in years, median & 63 & 8.75 \\
\hline Male, $n(\%)$ & $16(66.7)$ & \\
\hline Body mass index in $\mathrm{kg} / \mathrm{m}^{2}$, median & 27.7 & 7 \\
\hline $\begin{array}{l}\text { Glomerular filtration rate }>60 \mathrm{~mL} / \\
\text { minute per } 1.73 \mathrm{~m}^{2} \text { at time of } \\
\text { transplant, } n(\%)\end{array}$ & $13(54.2)$ & \\
\hline $\begin{array}{l}\text { Renal replacement therapy at time } \\
\text { of transplant, } n(\%)\end{array}$ & $4(16.7)$ & \\
\hline $\begin{array}{l}\text { Combined liver-kidney transplant, } \\
n(\%)\end{array}$ & $5(20.8)$ & \\
\hline $\begin{array}{l}\text { Hepatitis B virus co-infected, } \\
n(\%)\end{array}$ & 0 & \\
\hline $\begin{array}{l}\text { Hepatocellular carcinoma at time } \\
\text { of transplant, } n(\%)\end{array}$ & $11(45.8)$ & \\
\hline \multicolumn{3}{|l|}{ Immunosuppression, $n(\%)$} \\
\hline Tacrolimus & $18(75)$ & \\
\hline Cyclosporine & $6(25)$ & \\
\hline \multicolumn{3}{|l|}{ Genotype after transplant, $n(\%)$} \\
\hline $1 a$ & $19(79.2)$ & \\
\hline $1 a / 1 b$ & $1(4.2)$ & \\
\hline $1 b$ & $2(8.3)$ & \\
\hline 3 & $2(8.3)$ & \\
\hline \multicolumn{3}{|l|}{ Donor characteristics } \\
\hline Age, median & 30.3 & 10 \\
\hline Male, $n(\%)$ & $14(58.3)$ & \\
\hline BMI in $\mathrm{kg} / \mathrm{m}^{2}$, median & 26.7 & 5 \\
\hline $\begin{array}{l}\text { Hepatitis B core antibody } \\
\text { positive, } n\end{array}$ & 0 & \\
\hline Donor risk index ${ }^{\mathrm{a}}$, median & 1.1 & 0.18 \\
\hline Cold ischemia time in $\mathrm{m}$, median & 348 & 196 \\
\hline
\end{tabular}

${ }^{\mathrm{a}}$ Donor risk index calculated from http://gastro.cchmc.org/calculators/donorrisk-index/.

Abbreviations: BMI, body mass index; IQR, interquartile range.

ribavirin ranged from $600 \mathrm{mg}$ to $1200 \mathrm{mg}$ per day. Ribavirin drug levels were not monitored during treatment.

The virologic response in our cohort is described in Table 3. Twenty-three patients $(95.8 \%)$ had undetectable viral loads at 12 weeks after completing treatment (i.e. $S_{V R} R_{12}$ ). One patient with genotype $1 \mathrm{~b}$ was a nonresponder. One patient with genotype 1 a relapsed after achieving $S V_{12}$. Viral loads at 24 weeks after completing treatment (i.e. SVR 24 ) were available for 17 patients, all of whom had undetectable viral loads. Both the nonresponder and the patient who relapsed were treated with alterative DAA regimens with subsequent $\mathrm{SVR}_{12}$. Six weeks after undergoing the combined liver-kidney transplantation, the DAA nonresponder was initiated on sofosbuvir and ledipasvir. HCV genotype prior to and after the transplant was $1 \mathrm{~b}$. Prior to the transplant, the patient had failed therapy with an interferon-based regimen, and before starting treatment the HCV viral load was greater than $100,000,000 \mathrm{IU} / \mathrm{mL}$. Six weeks after initiation of the DAA therapy, the patient was noted to have a viral load of 
Table 2. Direct-acting antiviral therapy regimens

\begin{tabular}{lll}
\hline Regimen & $\begin{array}{l}\text { Number of } \\
\text { patients }\end{array}$ & $\begin{array}{l}\text { Length in } \\
\text { weeks }(n)\end{array}$ \\
\hline Ledipasvir + Sofosbuvir & 12 & $12(6)$ \\
& & $24(6)$ \\
$\begin{array}{l}\text { Ledipasvir + Sofosbuvir } \\
+ \text { Ribavirin }\end{array}$ & 8 & $12(5)$ \\
$\begin{array}{l}\text { Sofosbuvir + Velpatasvir } \\
\text { Daclatasvir + Sofosbuvir } \\
+ \text { Ribavirin }\end{array}$ & 1 & $24(3)$ \\
$\begin{array}{l}\text { Glecaprevir + } \\
\text { Pibrentasvir }\end{array}$ & 1 & $12(2)$ \\
\hline
\end{tabular}

$17,300,000 \mathrm{IU} / \mathrm{mL}$ and despite continued DAA therapy, the viral load remained persistently elevated. The patient was compliant with his antiviral regimen and NS5A resistance was not assessed. After 4 months of treatment and persistently elevated HCV viral load, DAA therapy was withdrawn. The patient was started on glecaprevir and pibrentasvir for 12 weeks with subsequent $\mathrm{SVR}_{12}$.

One patient developed HCV recurrence after achieving $\mathrm{SVR}_{12}$. The patient was HCV genotype $1 \mathrm{a}$, and sofosbuvir and ledipasvir were initiated at 16 weeks after the LT. The patient completed 24 weeks of treatment and achieved $\mathrm{SVR}_{12}$. Twelve weeks later, an HCV viral load was ordered in the setting of mildly elevated aminotransferases. The HCV viral load was $721,000 \mathrm{IU} / \mathrm{mL}$ and the patient was found to have NS5A resistance. Thirty-four weeks later, the patient was started on sofosbuvir, velpatasvir, and voxilaprevir for twelve weeks and achieved $S_{V R}$.

Two patients were treated with DAA therapy prior to the LT. One patient received 6 months of sofosbuvir and ledipasvir, and subsequently relapsed. Post-LT, the patient was started on sofosbuvir, ledipasvir, and ribavirin for 12 weeks, with achievement of $S R_{12}$. A second patient received sofosbuvir, velpatasvir, and ribavirin prior to the $L T$, however relapsed. Twenty-four days after the $\mathrm{LT}$, the patient was started on glecaprevir and pibrentasvir for twelve weeks, with subsequent $\mathrm{SVR}_{12}$.

A single recipient received a graft with F2 fibrosis. The indication for LT was HCV cirrhosis and hepatocellular carcinoma, and after receiving exception points the MELD-Na at time of transplant was 22. The genotype was 1 a and the

Table 3. Virologic response

\begin{tabular}{ll}
\hline & $\mathrm{SVR}_{12}, n(\%)$ \\
\hline All patients, $n=24$ & $23^{\text {a }}(95.8)$ \\
Ribavirin & \\
Patients treated with ribavirin, $n=9$ & $9(100)$ \\
Patients not treated with ribavirin, $n=15$ & $14(93.3)$ \\
DAA experienced & \\
DAA naïve, $n=22$ & $21(95.5)$ \\
DAA experienced, $n=2$ & $2(100)$
\end{tabular}

${ }^{a}$ One patient developed $\mathrm{HCV}$ recurrence after achieving $\mathrm{SVR}_{12}$. Abbreviation: DAA, direct-acting antiviral. patient received a 12-week course of sofosbuvir and ledipasvir, with achievement of $\mathrm{SVR}_{12}$.

All patients received induction immunosuppression with methylprednisolone followed by a 4-week prednisone taper and mycophenolate mofetil. Patients were also started on a calcineurin inhibitor and the majority $(75 \%)$ received tacrolimus. No episodes of rejection were noted. Drug levels were monitored on a regular basis and no significant interactions were noted between the DAAs and the immunosuppressants.

Five patients (20.8\%) developed significant adverse events during treatment. Pre- and posttreatment laboratory values are shown in Table 4. Acute kidney injury was observed in 2 cases. The first patient developed acute kidney injury while taking sofosbuvir in the setting of contrast exposure and several hypotensive episodes. In this patient, renal function returned to baseline with supportive care. The second patient developed acute kidney injury 4 months after LT, while taking tenofovir disoproxil concomitantly with sofosbuvir as suppressive therapy for hepatitis $B$. Renal function returned to baseline after discontinuation of tenofovir. Two patients developed significant anemia (hemoglobin $<8 \mathrm{gm} / \mathrm{dL}$ ) while on a ribavirin containing regimen. One patient required ribavirin dose reduction, and the second patient's hemoglobin stabilized after transfusion of 2 units of packed red blood cells. One patient developed acute monoarthritis in the setting of hyperuricemia. His symptoms improved with an intraarticular steroid injection and urate lowering therapy. No patients had evidence of early graft dysfunction or underwent re-transplantation.

\section{Discussion}

Over the past several years, DAAs have demonstrated proven efficacy and tolerability in the general population. ${ }^{8}$ More recently, studies have shown similar results in the post-liver transplant population. ${ }^{16-19}$ While DAAs are being used increasingly post-LT, there is limited data on the use of DAAs in HCV viremic patients receiving a graft from a HCV viremic donor. In this retrospective, multicenter study, HCV viremic patients were treated with DAAs after receiving a graft from a HCV viremic donor. DAAs proved to be efficacious and well tolerated in the vast majority of patients.

Table 4. Laboratory values before and after DAA therapy

\begin{tabular}{lll}
\hline Laboratory values & $\begin{array}{l}\text { Before } \\
\text { treatment }\end{array}$ & Posttreatment \\
\hline $\begin{array}{l}\text { Renal insufficiency as } \\
\text { GFR }<30 \mathrm{~mL} / \mathrm{min} / 1.73\end{array}$ & 0 & $1(4.1 \%)$ \\
$\mathrm{m}^{2}, n(\%)$ & \\
$\begin{array}{l}\text { Total bilirubin of }>1.4 \\
\text { mg/dL, } n(\%)\end{array}$ & 0 & 0 \\
$\begin{array}{l}\text { Hypoalbuminemia as } \\
\text { albumin }<3.5 \mathrm{gm} / \mathrm{dL},\end{array}$ & $4(16.6 \%)$ & $2(8.3 \%)$ \\
$n(\%)$ & \\
$\begin{array}{l}\text { Thrombocytopenia as } \\
\text { platelets }<150,000 / \mathrm{mcl}),\end{array}$ & $9(37.5 \%)$ & $5(20.38 \%)$ \\
$n(\%)$ & & \\
$\begin{array}{l}\text { ALT elevation as }>30 \mathrm{U} / \mathrm{L}, \\
n(\%)\end{array}$ & $11(45.8 \%)$ & $3(12.5 \%)$ \\
\hline
\end{tabular}

Abbreviations: ALT, alanine transaminase; DAA, direct-acting antiviral therapy; GFR, glomerular filtration rate. 
Two patients were infected with genotype 3 and were treated with sofosbuvir and ledipasvir. Though recent societal guidelines do not recommend sofosbuvir and ledipasvir in patients with genotype 3 , these patients were treated prior to the knowledge and availability of alternative regimens. Two patients who had received DAAs prior to LT and relapsed were successfully treated post-LT, with achievement of SVR 12 .

The timing of antiviral therapy was determined by individual hepatologists after a careful review of several factors, including normalization of renal function, a stable immunosuppression regimen, and obtaining insurance approval for DAA therapy. Thus far, the timing of DAA therapy has not been well defined in the posttransplant population. In theory, early initiation of DAA therapy, while viral loads remain low, may mitigate the risk of certain complications such as fibrosing cholestatic hepatitis. In our study, however, the level of viremia did not influence when therapy was initiated.

In the pre-DAA era, several studies evaluated the efficacy and safety of interferon-based regimens post-LT. These regimens were associated with significant adverse events and suboptimal efficacy. One large study assessed 113 patients post-LT and observed SVR in $38 \%$ of patients. ${ }^{20}$ Over the past decade, several studies have evaluated the safety and efficacy of DAAs in HCV patients post-LT. The majority of patients in these studies are genotype 1 , and $\mathrm{SVR}_{12}$ was observed in greater than $90 \%$ of patients. ${ }^{21-23}$ Many of these studies were retrospective in nature and with variable time between LT and initiation of treatment. Most studies started antiviral treatment more than 3 years after transplant; however, several started treatment within 6 months of transplant. ${ }^{24,25}$ In our cohort, the mean time from transplant to initiation of therapy was 128 days.

None of the donors in our study had advanced hepatic fibrosis at the time of transplant. Donors with F3 or F4 hepatic fibrosis were not considered. Though changes in fibrosis were not objectively measured during treatment, others have suggested that early initiation of DAAs may result in regression of fibrosis in the graft. ${ }^{26}$ Our experience indicates that early initiation of DAA therapy in the posttransplant population is well tolerated and does not cause significant changes in the patients' immunosuppression regimens. Treating patients with HCV on the waiting list may be a cost-effective strategy ${ }^{27}$ but it could decrease access to organs from HCV viremic donors. Though patients with a low MELD-Na score may benefit from early DAA treatment, ${ }^{28}$ we believe delaying treatment in patients with decompensated cirrhosis until after LT increases access to potential donors and decreases time on the waiting list. We add to the growing body of evidence that antiviral therapy should be initiated soon after LT, as it is well tolerated and may modify the course of fibrosis in the graft. ${ }^{28}$

The majority of the studies evaluating the use of DAAs in the post-LT population included ribavirin as part of the antiviral regimen. In the past, ribavirin has been a critical component of most antiviral regimens, however it may add to the cost of treatment and be associated with significant adverse events. More recently, it has been suggested that HCV-positive transplant recipients can be treated with sofosbuvir and ledipasvir without ribavirin irrespective of treatment duration and degree of liver fibrosis. Most (62.5\%) of our patients were treated with ribavirin-free regimens and demonstrated similar efficacy to those treated with ribavirin. Additionally, two patients developed significant anemia requiring either adjustment or discontinuation of ribavirin. This finding, when taken in the context of other recent studies, questions the requirement and benefit of ribavirin when treating patients with DAAs.
The attitude and approach towards HCV-positive grafts have evolved over the past decade. Between 2005 and 2015, Bowring and colleagues ${ }^{4}$ observed that though HCV-positive grafts are being increasingly utilized, they are nearly twice as likely to be discarded as compared to an HCV-negative graft. In our cohort, the average MELD-Na score was 21 at the time of transplant. In addition, the median time on the transplant waitlist was 81 days and the average was 127 days. While attitudes towards HCV-positive grafts have changed over the past 2 decades, these organs remain under-utilized. In the DAA era, the introduction of grafts from HCV viremic donors may potentially expand the donor pool and decrease time on the waitlist, therefore increasing the number of patients who ultimately undergo LT. Though further research is required, the results from our study may be extrapolated to support the growing interest in the transplantation of $\mathrm{HCV}$ viremic organs into HCV-negative recipients.

This retrospective study had several limitations. The cohort was limited to 24 patients, and due to the lack of guidelines at the time, treatment regimens and durations were heterogeneous and ultimately determined by the individual hepatologist. This led to a variety or regimens that were used, for durations ranging from 12 to 24 weeks. Also, no patients, except for one who relapsed, underwent NS5A resistance testing prior to initiation of DAA therapy.

As the landscape of organ transplantation shifts to expanding the donor pool and transplanting grafts from HCV viremic donors into HCV viremic recipients, the early initiation of DAAs in these patients appears to be effective and well tolerated. In this multicenter, retrospective study, we observed that the use of DAAs in HCV viremic patients who receive a graft from a HCV viremic donor was well tolerated and efficacious. These findings suggest that in the era of DAAs, there is the potential to further expand the donor pool and to decrease the waiting time prior to transplantation.

\section{Conflict of interest}

The authors have no conflict of interests related to this publication.

\section{Author contributions}

Designed and conceptualized the study (NK, KAK, KVNM, CL, $\mathrm{DR}, \mathrm{JMV}, \mathrm{SE}, \mathrm{AT}$, and $\mathrm{XBZ}$ ), collected data (NK and GF), wrote the paper (NK) and critically revised the paper (KAK, KVNM, $C L$, DR, JMV, SE, AT, and XBZ).

\section{References}

[1] Kim WR, Lake JR, Smith JM, Skeans MA, Schladt DP, Edwards EB, et al. OPTN/SRTR 2015 Annual Data Report: Liver. Am J Transplant 2017;17: 174-251. doi: 10.1111/ajt.14126.

[2] Taylor J, Cox-North P, Landis CS. Management of post-liver transplant recurrence of hepatitis C. Drugs 2016;76:1711-1717. doi: 10.1007/s40265-0160658-0.

[3] Akamatsu N, Sugawara Y. Liver transplantation and hepatitis C. Int J Hepatol 2012;2012:686135. doi: 10.1155/2012/686135.

[4] Bowring MG, Kucirka LM, Massie AB, Luo X, Cameron A, Sulkowski M, et al. Changes in utilization and discard of hepatitis C-infected donor livers in the recent era. Am J Transplant 2017;17:519-527. doi: 10.1111/ajt.13976.

[5] Northup PG, Argo CK, Nguyen DT, McBride MA, Kumer SC, Schmitt TM, et al. Liver allografts from hepatitis $C$ positive donors can offer good outcomes in hepatitis C positive recipients: a US National Transplant Registry analysis. Transpl Int 2010;23:1038-1044. doi: 10.1111/j.1432-2277.2010.01092.x. 
[6] Chung RT, Davis GL, Jensen DM, Masur H, Saag MS, Thomas DL, et al. Hepatitis $C$ guidance: AASLD-IDSA recommendations for testing, managing, and treating adults infected with hepatitis C virus. Hepatology 2015;62: 932-954. doi: 10.1002/hep.27950.

[7] Burton JR Jr, O'Leary JG, Verna EC, Saxena V, Dodge JL, Stravitz RT, et al. A US multicenter study of hepatitis $C$ treatment of liver transplant recipients with protease-inhibitor triple therapy. J Hepatol 2014;61:508-514. doi: 10. 1016/j.jhep.2014.04.037.

[8] Asselah T, Marcellin P. Interferon free therapy with direct acting antivirals for HCV. Liver Int 2013;33:93-104. doi: 10.1111/liv.12076.

[9] Lynch SM, Wu GY. Hepatitis C virus: A review of treatment guidelines, costeffectiveness, and access to therapy. J Clin Transl Hepatol 2016;4:310-319. doi: $10.14218 /$ JCTH.2016.00027.

[10] Felmlee DJ, Coilly A, Chung RT, Samuel D, Baumert TF. New perspectives for preventing hepatitis $C$ virus liver graft infection. Lancet Infect Dis 2016;16: 735-745. doi: 10.1016/S1473-3099(16)00120-1.

[11] Ueda Y, Ikegami T, Akamatsu N, Soyama A, Shinoda M, Goto R, et al. Treatment with sofosbuvir and ledipasvir without ribavirin for 12 weeks is highly effective for recurrent hepatitis $C$ virus genotype 1 b infection after living donor liver transplantation: a Japanese multicenter experience. J Gastroenterol 2017;52:986-991. doi: 10.1007/s00535-017-1310-9.

[12] Pungpapong S, Aqel B, Leise M, Werner KT, Murphy JL, Henry TM, et al. Multicenter experience using simeprevir and sofosbuvir with or without ribavirin to treat hepatitis $C$ genotype 1 after liver transplant. Hepatology 2015; 61:1880-1886. doi: 10.1002/hep.27770.

[13] Mitchell O, Gurakar A. Management of hepatitis C post-liver transplantation: A comprehensive review. J Clin Transl Hepatol 2015;3:140-148. doi: 10. 14218/JCTH.2015.00005.

[14] Hashimoto $\mathrm{K}$, Miller C. The use of marginal grafts in liver transplantation. J Hepatobiliary Pancreat Surg 2008;15:92-101. doi: 10.1007/s00534-0071300-z.

[15] Marroquin CE, Marino G, Kuo PC, Plotkin JS, Rustgi VK, Lu AD, et al. Transplantation of hepatitis C-positive livers in hepatitis C-positive patients is equivalent to transplanting hepatitis C-negative livers. Liver Transpl 2001; 7:762-768. doi: 10.1053/jlts.2001.27088.

[16] Bushyhead D, Goldberg D. Use of hepatitis C-positive donor livers in liver transplantation. Curr Hepatol Rep 2017;16:12-17. doi: 10.1007/s11901017-0327-0.

[17] Charlton M, Gane E, Manns MP, Brown RS Jr, Curry MP, Kwo PY, et al. Sofosbuvir and ribavirin for treatment of compensated recurrent hepatitis $C$ virus infection after liver transplantation. Gastroenterology 2015;148:108-117. doi: $10.1053 /$ j.gastro.2014.10.001
Kapila N. et al: LT with HCV viremic recipients and donors

[18] Coilly A, Fougerou-Leurent C, de Ledinghen V, Houssel-Debry P, Duvoux C, Di Martino $\mathrm{V}$, et al. Multicentre experience using daclatasvir and sofosbuvir to treat hepatitis C recurrence - The ANRS CUPILT study. J Hepatol 2016;65: 711-718. doi: 10.1016/j.jhep.2016.05.039.

[19] Kwo PY, Mantry PS, Coakley E, Te HS, Vargas HE, Brown R Jr, et al. An interferon-free antiviral regimen for HCV after liver transplantation. N Engl J Med 2014;371:2375-2382. doi: 10.1056/NEJMoa1408921.

[20] Roche B, Sebagh M, Canfora ML, Antonini T, Roque-Afonso AM, Delvart $V$, et al. Hepatitis $C$ virus therapy in liver transplant recipients: response predictors, effect on fibrosis progression, and importance of the initial stage of fibrosis. Liver Transpl 2008;14:1766-1777. doi: 10.1002/It.21635.

[21] Pillai AA, Maheshwari R, Vora R, Norvell JP, Ford R, Parekh S, et al. Treatment of HCV infection in liver transplant recipients with ledipasvir and sofosbuvir without ribavirin. Aliment Pharmacol Ther 2017;45:1427-1432. doi: 10. 1111/apt.14059.

[22] Saxena V, Khungar V, Verna EC, Levitsky J, Brown RS Jr, Hassan MA, et al. Safety and efficacy of current direct-acting antiviral regimens in kidney and liver transplant recipients with hepatitis C: Results from the HCV-TARGET study. Hepatology 2017;66:1090-1101. doi: 10.1002/hep.29258.

[23] Kwok RM, Ahn J, Schiano TD, Te HS, Potosky DR, Tierney A, et al. Sofosbuvir plus ledispasvir for recurrent hepatitis $C$ in liver transplant recipients. Liver Transpl 2016;22:1536-1543. doi: 10.1002/It.24614.

[24] Leroy V, Dumortier J, Coilly A, Sebagh M, Fougerou-Leurent C, Radenne S, et al. Efficacy of sofosbuvir and daclatasvir in patients with fibrosing cholestatic hepatitis C after liver transplantation. Clin Gastroenterol Hepatol 2015; 13:1993-2001.e2. doi: 10.1016/j.cgh.2015.05.030.

[25] Forns X, Charlton M, Denning J, McHutchison JG, Symonds WT, Brainard D, et al. Sofosbuvir compassionate use program for patients with severe recurrent hepatitis C after liver transplantation. Hepatology 2015;61:1485-1494. doi: 10.1002/hep.27681.

[26] Martini S, Salizzoni M, David E, Tandoi F, Fonio P, Delsedime L, et al. Favorable short-term outcome of hepatitis $C$ virus-positive liver graft with bridging fibrosis: A plea for very early viral eradication. Hepatology 2017;65:21162118. doi: 10.1002/hep.28978.

[27] Ahmed A, Gonzalez SA, Cholankeril G, Perumpail RB, McGinnis J, Saab S, et al. Treatment of patients waitlisted for liver transplant with all-oral directacting antivirals is a cost-effective treatment strategy in the United States. Hepatology 2017;66:46-56. doi: 10.1002/hep.29137.

[28] Samur S, Kues B, Ayer T, Roberts MS, Kanwal F, Hur C, et al. Cost Effectiveness of pre- vs post-liver transplant hepatitis $C$ treatment with direct-acting antivirals. Clin Gastroenterol Hepatol 2018;16:115-122.e10. doi: 10. 1016/j.cgh.2017.06.024. 